\title{
Using remote sensing for monitoring the dynamic of soil organic carbon concentration in Lake Valencia basin, Venezuela, based on Landsat 8 data
}

\author{
Israel Jose Arias Govín ${ }^{1 *}$,Elena V. Stanis ${ }^{1}$, Elena $N$. Latushkina ${ }^{1}$, and Aigul Ospanova ${ }^{1}$ \\ ${ }^{1}$ Peoples' Friendship University of Russia (RUDN University), Faculty of Ecology, 6 Miklukho- \\ Maklaya St, Moscow, 117198, Russian Federation
}

\begin{abstract}
Maintaining or increasing SOC concentration is fundamental for reducing the effects of global warming and increasing soil productivity. In this paper, a method based on Landsat 8 OLI products was developed for qualitatively monitoring in the Lake Valencia basin (Venezuela) the dynamic of SOC concentration between the years 2013 to 2018. The developed method uses the Green (B3), NIR (B5) and SW1 (B6) bands of Landsat 8 OLI sensor for detecting changes in the spectral signatures of bare soils that indicate possible variations in their concentrations of SOC. It was found that for the study period, the Lake Valencia basin soils do not present spectral features of significant variation in SOC concentration. An area of $8.61 \mathrm{Km}^{2}(0.3 \%$ of the study area) was identified as a zone with a possible reduction of SOC concentration. In case of insufficient data for developing remote sensing based predictive models, the proposed method allows qualitatively monitoring and categorizing the dynamic of SOC concentration and identifying areas with spectral features of a possible variation in SOC concentration.
\end{abstract}

\section{Introduction}

Soil organic carbon (SOC) represents $69.8 \%$ of organic C in the global carbon cycle in the biosphere [1]. The total amount of organic $\mathrm{C}$ stored in soils has been estimated by different methods $[2,3]$ and it is considered to be close to $1500 \mathrm{Pg}$ at $1 \mathrm{~m}$ depth $[1,4]$. This means more $\mathrm{C}$ than the content between the atmosphere and the terrestrial vegetation [5] representing the largest world reserve of terrestrial $\mathrm{C}[6,7]$. Therefore, $\mathrm{C}$ sequestration in soils could contribute significantly to reducing global warming levels [8]. On the other hand, the increase in SOC directly influences the improvement of the structure, nutrient retention, physical and chemical properties of soils, and consequently an increase of soil fertility [9].

Due to the importance of the SOC, the FAO technical report states that keeping the pool of COS in balance or increasing the SOC content towards the optimum level for the local environment can contribute to achieving the Sustainable Development Goals [10]. For

\footnotetext{
* Israel Jose Arias Govin: israelgovin@gmail.com
} 
achieving this, it is essential to develop profitable tools and methods applicable to different types of soil allowing regular monitoring and mapping of SOC variations. The above represents a challenge because the spatial distribution of the SOC is not uniform across large surfaces and at different depths of the ground [10]. Besides, variations in the concentration and spatial distribution of SOC in soils are controlled partially by spatially and temporally variable factors such as environmental conditions, vegetation cover, land use, and soil edaphic characteristics [11-13].

Until the date, there are no standardized methods for determining the concentration of SOC [10]. Most of the traditional methods based on the oxidation of $C$ involve high costs in time and resources for collecting, preparing, and processing the soil sample [14]. Satellite remote sensing techniques have been used for developing predictive models of SOC concentration in different types of soil [15-18]. The results obtained in these investigations demonstrate that using satellite remote sensors for estimating the SOC concentration is feasible. Nevertheless, for developing statistical models it is essential to calibrate them with data analysed in laboratory conditions. This is because the spectral response of the soils is affected not only by SOC concentration but also by its chemical and physical characteristics [19]. The extrapolation of predictive models to different areas for which they were developed would imply considerable errors in the prediction of SOC concentration [20].

In this study case, due to insufficient data for developing a statistical model for estimating SOC concentration in the Lake Valencia basin (Venezuela), it will be developed a method based on Landsat 8 OLI products for qualitatively analysing the dynamic of SOC concentration between the years 2013 to 2018 in the study area.

\section{Study area}

Lake Valencia is the most important natural endorheic lake of South America [21] with an approximate surface of $344 \mathrm{~km}^{2}$. The basin of Lake Valencia covers an area of approximately $3000 \mathrm{~km}^{2}$. Figure 1 shows the location of the study area and land use classification.

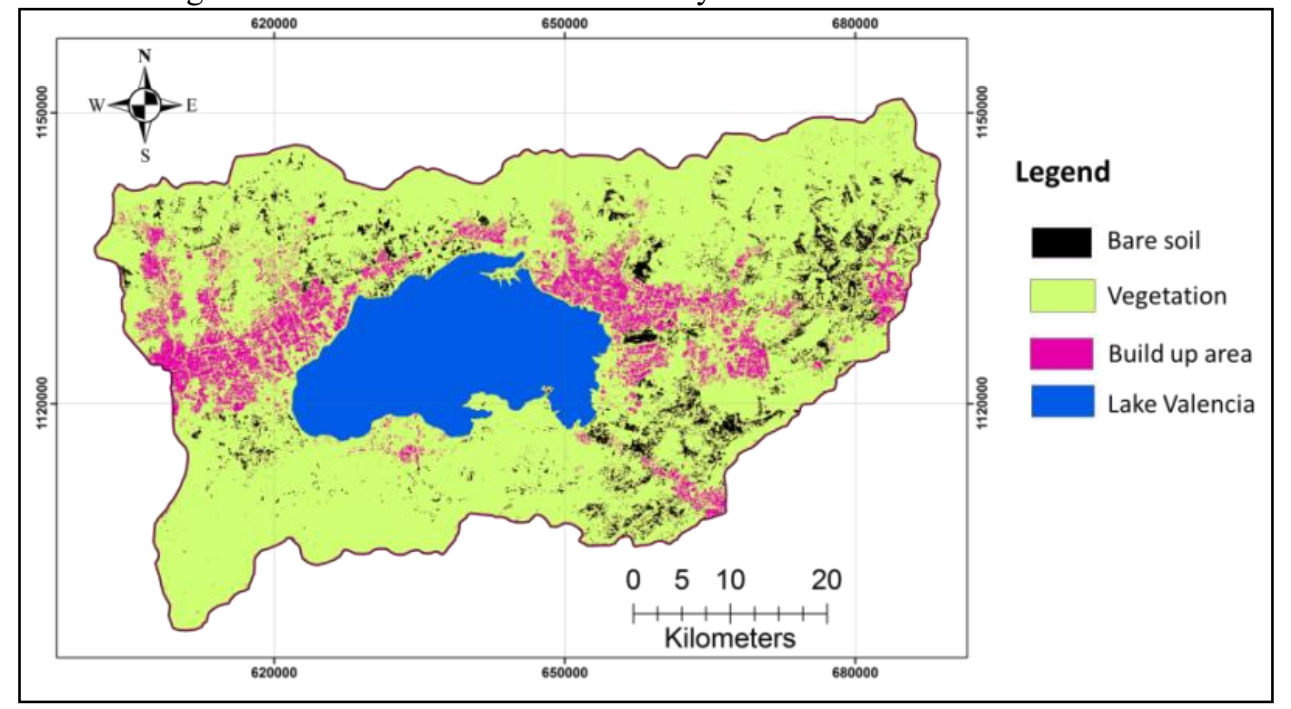

Fig. 1. Location of the study area and land use classification

The predominant soil type belongs to the category of inceptisols, with medium texture. Mollisols soils are medium textured with high organic matter content. In the northwestern 
region of the basin, oxisol soils with high iron concentrations are scarcely found [22-24]. Figure 3 shows the soil types distribution in the study area.

\section{Materials and methods}

Several studies have characterized the spectral behaviour of soils $[14,19,20,25,26]$. The results of these investigations agree that soils with higher SOC concentration have lower reflectance values in their spectral signature compared to the reflectance values of the spectral signature of soils with lower SOC concentration. Given the above considerations, for the specific same sample of soil, an increase in the reflectance of its spectral signature could be largely associated with a decrease in their SOC concentration.

Figure 2 shows the spectral profile of 5 types of soils with different SOC concentration based on the studies by Shi et al. [19], and modified according to the spectral bands of the Landsat 8 OLI sensor. Figure 2 shows that the decrease in SOC concentration is characterized by a significant increase in the reflectances of the bands Green $(561 \mathrm{~nm})$, NIR $(865 \mathrm{~nm})$ and SW1 $(1609 \mathrm{~nm})$, the bands corresponding to Blue $(483 \mathrm{~nm})$, Red $(655 \mathrm{~nm})$ and SW2 (2201nm) do not have a relevant impact on the variation of the spectral signature associated with an increase or decrease in the SOC concentration. Following this, the bands Green (B3), NIR (B5) and SW1 (B6) were selected for developing the method applied. The method developed consists of 4 stages: a) multispectral image pre-processing; b) selection of bare soil points; c) determination of the spectral signatures variations at the selected points; and d) interpolation and obtaining the distribution map of the SOC concentration variation during the study period.

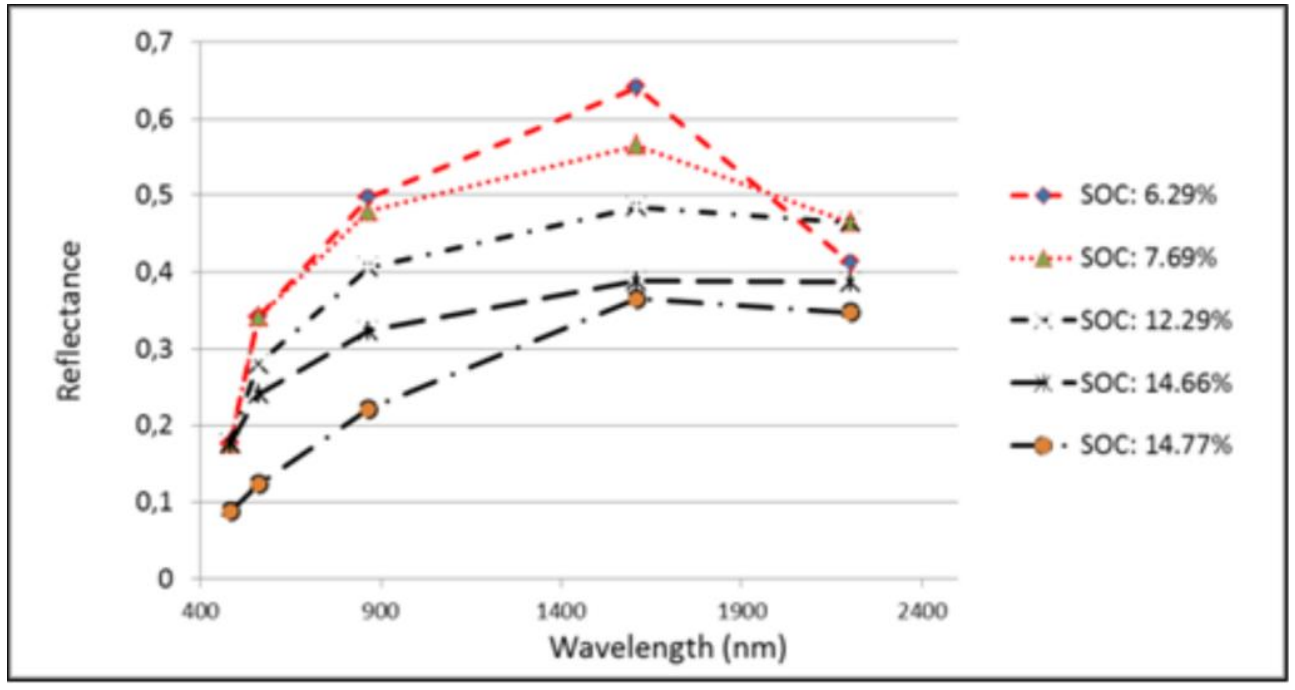

Fig. 2. Spectral profiles of soils with different SOC concentration adapted to the spectral bands of the Landsat 8 OLI sensor. Modified from Shi et al. [19]

In the first stage, four images of the Landsat 8 OLI sensor were used. Two images with the lowest cloud coverage percentage were selected for each year of study (2013 and 2018) to cover the area of the basin. The images correspond to the month of November (dry season) to guarantee the lowest level of humidity in the soils. The top of atmosphere (TOA) reflectance values were obtained according to the procedures described in the Landsat 8 user manual [27]. Atmospheric correction for obtaining the top of canopy (TOC) reflectance values, was performed by applying the DOS method developed by Chavez [28]. 
In the second stage, the coincident points of bare soils in the two years studied were selected. Initially, to identify areas of bare soil without vegetation, water, and cloudiness, a mask was created by applying the NDSI (Normalized Difference Soil Index) [29] in both scenes using Equation 1. In Equation 1, NDSI is the Normalized Difference Soil Index, B5 and B6 are the TOC reflectance raster of the NIR and SW1 bands. Then, the classification of the NDSI rasters obtained was performed and polygons were generated for bare soil areas and build-up areas. Points were created randomly within the corresponding polygons to the bare soil area. A total of 2475 coincident points for both years were selected. Figure 3 shows the spatial distribution of the selected bare soil points.

$$
N D S I=(B 6-B 5) /(B 6+B 5)
$$

In the third stage, the variations of the spectral profiles were obtained at the selected points. For this, a mask was created by applying Equation 2 using the TOC reflectances bands B3, B5, and B6. The pixels of the raster obtained have values 1 (one) or 0 (zero). Pixels with a value of 1 correspond to the points whose spectral signatures showed an increase in reflectance between 2013 and 2018. A total of 163 points that are shown in Figure 3 were identified.

$$
\left(\left(\left(B 3_{2013}-B 3_{2018}\right)<0\right)+\left(\left(B 5_{2013}-B 5_{2018}\right)<0\right)+\left(\left(B 6_{2013}-B 6_{2018}\right)<0\right)\right) / 3
$$

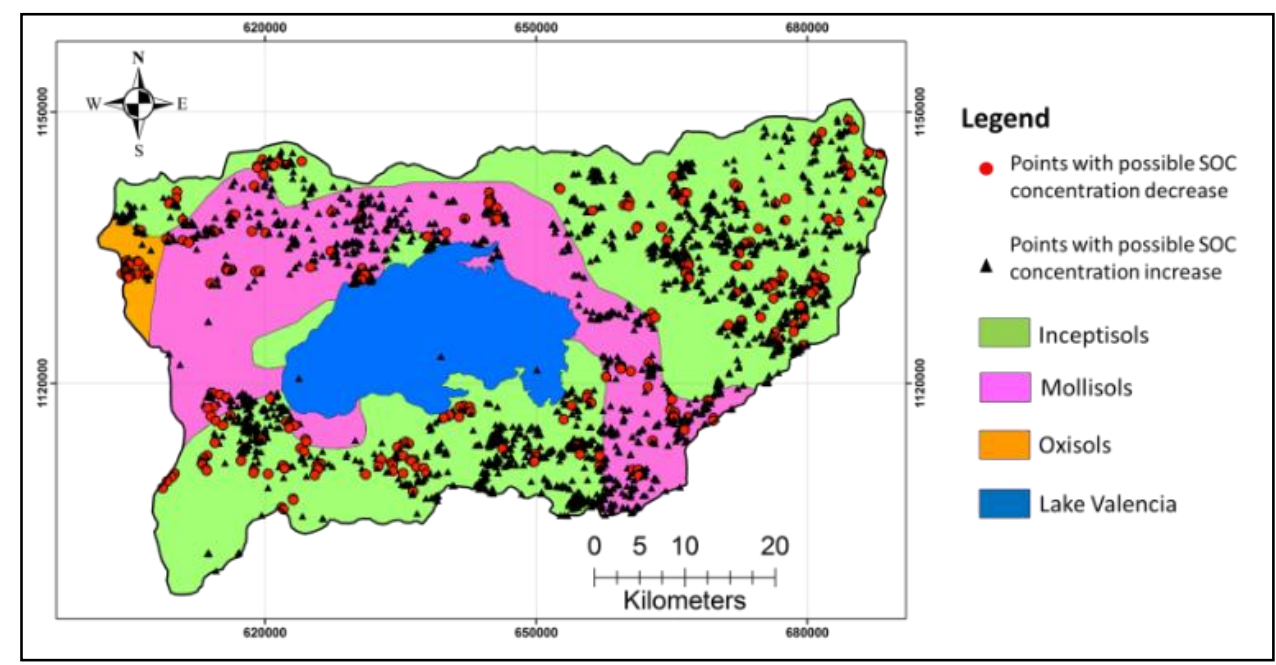

Fig. 3. Soil types and bare soil points distribution

In the fourth stage, the method applied for classifying the SOC concentration variation was based on the comparison of the area under the reflectance curve between the wavelengths $0.561 \mu \mathrm{m}$ (B3) and 1,609 $\mu \mathrm{m}$ (B6) for each point between the years 2013 and 2018. For determining the area under the curve, the model presented in Equation 4 was developed, where $A_{i-j}$ is the area under the reflectance curve for each point " $i$ " in the year " $j$ " and B3, B5 and B6 the TOC reflectance rasters of the Green, NIR and SW1 bands respectively. Equation 4 was obtained from Equation 3 using the wavelength values of the corresponding bands for the Landsat 8 sensor. In Equation 3, $\mathrm{L}_{\mathrm{B} 3}, \mathrm{~L}_{\mathrm{B} 5}$, and $\mathrm{L}_{\mathrm{B} 6}$ are the wavelengths of bands $\mathrm{B} 3, \mathrm{~B} 5$, and $\mathrm{B} 6$ respectively. Two rasters corresponding to the values of $\mathrm{A}_{\mathrm{i}-\mathrm{j}}$ of every point were obtained, one for the year $2013\left(\mathrm{~A}_{\mathrm{i}-2013}\right)$ and the other for the year $2018\left(\mathrm{~A}_{\mathrm{i}-2018}\right)$.

$$
\begin{gathered}
\mathrm{A}_{\mathrm{i}-\mathrm{j}}=0.5 *\left(\left(\mathrm{~L}_{\mathrm{B} 5}-\mathrm{L}_{\mathrm{B} 3}\right) *(\mathrm{~B} 5+\mathrm{B} 3)+\left(\mathrm{L}_{\mathrm{B} 6}-\mathrm{L}_{\mathrm{B} 5}\right) *(\mathrm{~B} 6+\mathrm{B} 5)\right) \\
A_{i-j}=0.152 *(B 3)+0.524 *(B 5)+0.372 *(B 6)
\end{gathered}
$$


Six (6) categories were established to qualitatively classify the variation of the SOC concentration according to the difference of the areas under the reflectance curve for the same point between 2013 and 2018 using Equation 5. In Equation 5, $\mathrm{V}_{\mathrm{SOC}}$ is the grade of variation of the SOC concentration, $\mathrm{A}_{\mathrm{i}-2013}$, and $\mathrm{A}_{\mathrm{i}-2018}$ are the raster previously obtained. The categories used were the following: a) $\mathrm{V}_{\mathrm{SOC}}<-0.2$, severe decrease; b) $-0.2 \leq \mathrm{V}_{\mathrm{SOC}}<-0.1$, moderate decrease; c) $-0.1 \leq \mathrm{V}_{\mathrm{SOC}}<0$, no decrease or slight decrease; d) $0 \leq \mathrm{V}_{\mathrm{SOC}}<0.1$, no increase or slight increase; e) $0.1 \leq \mathrm{V}_{\mathrm{SOC}} \leq 0.2$, moderate increase; and f) $\mathrm{V}_{\mathrm{SOC}}>0.2$, high increase. As a result, the raster corresponding to the variation of the SOC concentration for each point selected in stage 2 was obtained. Finally, after obtaining the $\mathrm{V}_{\text {SOC }}$ values for each point, the IDW interpolation was performed to obtain the distribution and categorization map of the SOC concentration dynamic in the study area between the years 2013 and 2018.

$$
V_{S O C}=\left(A_{i-2013} / A_{i-2018}\right)-1
$$

\section{Results and discussion}

For validating the results of the method developed in the study area, the variation of the spectral signatures of 1610 points (65\% of the bare soil points) was checked. The validation confirmed the spectral behaviors of possible decrease or increase of the SOC concentration. Figure 4 shows the spectral signatures of six characteristic points within the zones of the SOC concentration variation categories established: a) severe decrease; b) moderate decrease; c) no decrease or slight decrease; d) no increase or slight increase; e) moderate increase; and f) high increase.

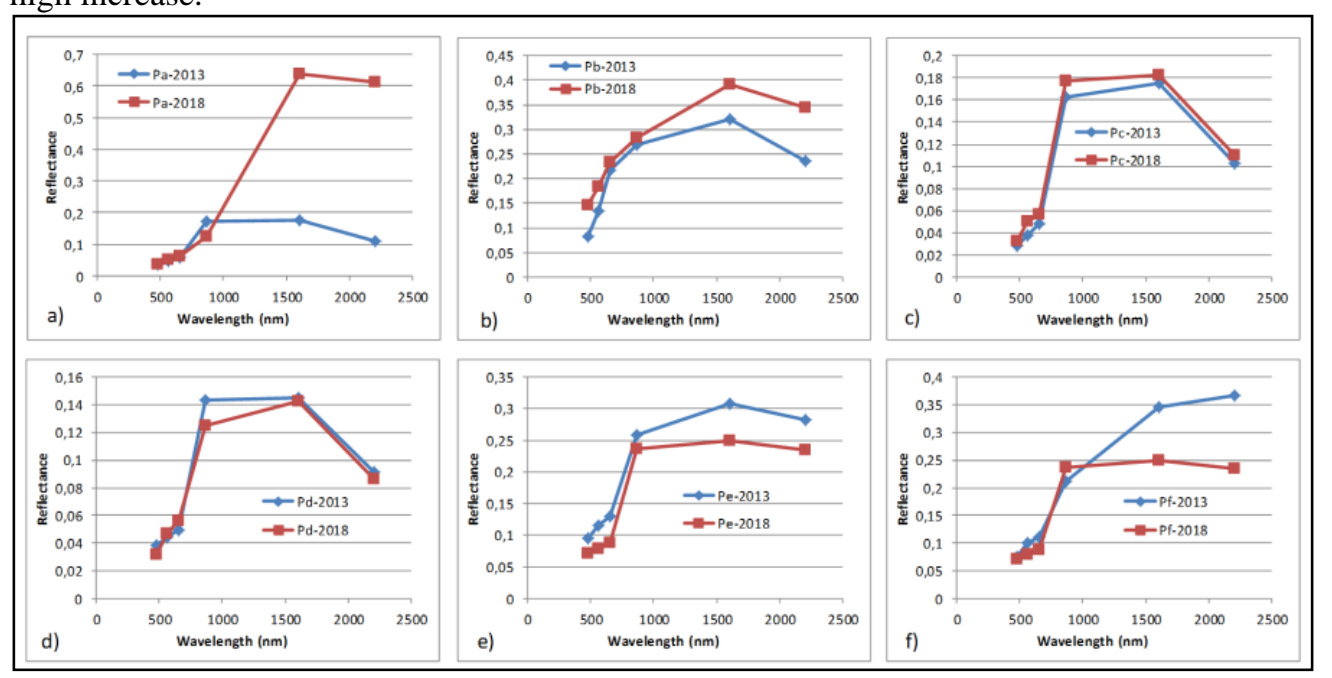

Fig. 4. Spectral signatures of characteristic points for every SOC variation categories established

Figure 5 shows the distribution map of the SOC concentration variations in the study area. The results show that for the study period, the soils of the Lake Valencia basin do not preset relevant signs of SOC concentration decrease. $96.57 \%$ of the total area $\left(2919.86 \mathrm{~km}^{2}\right)$ of the basin did not present variations in their soils spectral signatures that indicate significant increases or decreases in the concentration of SOC. The $3.13 \%$ of the study area $\left(91.59 \mathrm{~km}^{2}\right)$ corresponds to zones with possible increases of SOC concentration and only $0.3 \%\left(8.61 \mathrm{Km}^{2}\right)$ showed signs of a possible reduction of SOC concentration. 


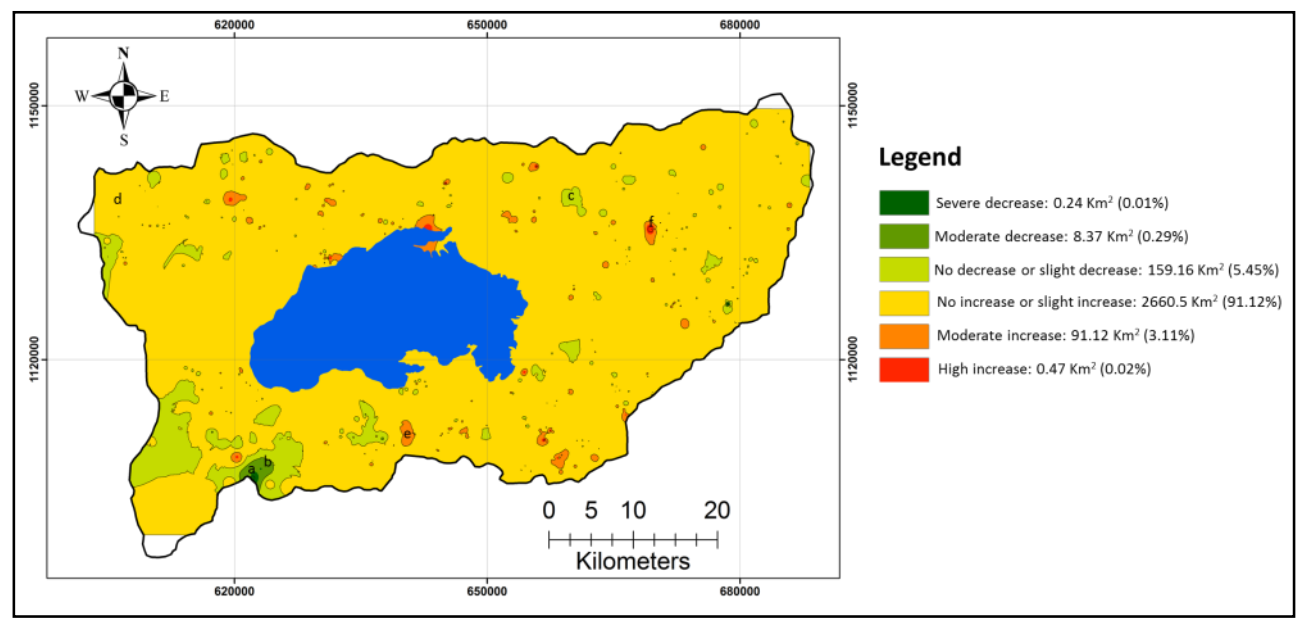

Fig. 5. Distribution map of the SOC concentration variation in the study area from 2013 to 2018

\section{Conclusions}

The developed method is based on the relationship between the spectral behavior of soils and its concentration of SOC. It was found that using Green (B3), NIR (B5) and SW1 (B6) bands of the Landsat 8 OLI sensor is feasible for detecting changes in the spectral signatures of bare soils that indicate possible variations in the concentration of SOC. The proposed method allows qualitatively monitoring the temporal and spatial dynamics of the SOC and identifying the areas with signs of possible decrease or increase in SOC as well as to categorize the degree of variation. The NDSI allows discriminating areas of vegetation, water bodies, cloudiness, and shadows, highlighting areas of bare soil and build-up areas. Its use is recommended together with visual review for a more accurate selection of points corresponding to bare soil. Adaptation of the method for using with other remote sensors should consider the adjustment of Equation 3 according to the wavelength values for the Green, NIR and SW 1 bands of the sensor to be used. The main limitation of the method is that it is not able to quantitatively estimate the SOC concentration.

\section{References}

1. FAO. Soil carbon sequestration for improved land management. 96, p.58, (2001).

2. W. M. Post, et al. Nature. 298, 156-159, (1982)

3. R. S. Swift. J. Soil Sci., 166, 858-871, (2001)

4. W. H. Schlesinger. Nature, 348, 232-234 (1990)

5. FAO-GTIS. Status of the World's Soil Resources, (2015)

6. E. Jobbágy, R. Jackson. Ecol. Appl., 10, (2000).

7. J. Scharlemann, E. Tanner, R. Hiederer, V. Kapos. Carbon Manag., 5, 81-91, (2014)

8. T.W. Crowther et al. Nature, 540, 104-108, (2016)

9. T. Ontl, L. Schulte. Nat. Educ. Knowl., 3, 35-45, (2012)

10. FAO. Soil Organic Carbon: the hidden potential, (2017)

11. J. Bouma. J. Environ. Qual., 26(1), 26-31, (1997)

12. D. Karlen, et al. S. Sc. Soc. Am. J., 61(1), 4-10, (1997)

13. Y. Guo, R. Amundson, P. Gong, Q. Yu. Soil Sci. Soc. Am. J., 70, 590-600 (2006)

14. A. Gholizadeh, et al. Appl. Spectrosc., 67(12),1349-1362, (2013)

15. D. Rogge, et al. Remote Sens. Environ, 205, 1-17, (2018) 
16. F. Castaldi, et al. Remote Sensing, 10 (2), 153, (2018)

17. T. Angelopoulou, et al. Remote Sensing, 11(6), 676, (2019)

18. F. Castaldi, et al. Remote Sensing., 11, 2121, (2019)

19. Z. Shi, et al. Science China. Earth Sciences, 57(7), 1671-1680, (2014)

20. H. Bartholomeus, et al. Geoderma, 145, 28-36, (2008)

21. M. W. Binford. Ecol. Monogr., 52(3), 307-333, (1982)

22. INE. Informe Geoambiental, Estado Carabobo. INE, Rep. B. Venezuela, (2011)

23. INE. Informe Geoambiental, Estado Aragua. INE, Rep. B. Venezuela, (2011)

24. L. Mogollón, J. Comerna. Suelos de Venezuela. PDVSA, (1995)

25. E. Stoner, M. Baumgardner. Tech. Rep., Purdue Univ., (1982).

26. T. Henderson, et al. Soil Sci. Soc. A. J., 53, 1778-1784, (1989).

27. USGS. LANDSAT 8 (L8) Data users handbook. Version 3.0 - LSDS-1574, (2018)

28. J. Chavez. Remote Sens. Environ., 24, 459-479, (1988)

29. A. Rogers, M.S., Kearney. Int. J. of Rem. Sens., 25(12), 2317-2335, (2004) 